\title{
Proteomics
}

\section{Die Proteinausstattung eines einzelnen pflanzlichen Mitochondriums}

\author{
TATJANA HILDEBRANDT ${ }^{1}$, ETIENNE MEYER ${ }^{2}$, MARKUS SCHWARZLÄNDER ${ }^{3}$ \\ ${ }^{1}$ INSTITUT FÜR PFLANZENGENETIK, UNIVERSITÄT HANNOVER \\ 2 INSTITUTSBEREICH PFLANZENPHYSIOLOGIE, UNIVERSITÄT HALLE-WITTENBERG \\ ${ }^{3}$ INSTITUT FÜR BIOLOGIE UND BIOTECHNOLOGIE DER PFLANZEN, UNIVERSITÄT \\ MÜNSTER
}

\section{The structure and function of mitochondria have been characterized with increasing precision. How the protein inventory defines the characteristics of the organelle remains insufficiently understood, however. Recently we devised a quantitative proteomic approach to estimate the copy numbers of proteins in a single plant mitochondrion, as physical operational unit in the cell. We illustrate how such a simple thought experiment can give fascinating insights into how a mito- chondrion works.}

DOI: $10.1007 / \mathrm{s} 12268-021-1674-5$

(C) Die Autorinnen und Autoren 2021

Mitochondrien sind tief verwurzelt in der Evolution komplexen Lebens. Sie übernehmen zentrale Aufgaben im zellulären Energiestoffwechsel und in der Signaltransduktion. Unser aktuelles Verständnis von Mitochondrien fällt größtenteils in zwei Kategorien, die sich während der vergangenen Jahrzehnte weitgehend parallel entwickelt haben:
Einerseits konnten die biochemischen und physiologischen Funktionen von Mitochonseits haben wir tiefe Einsichten in die Organisation und Struktur von Mitochondrien in der Zelle gewonnen, vornehmlich dank zellbiologisch-mikroskopischer Verfahren. Diese beiden Blickwinkel unterscheiden sich drien im Detail untersucht werden. Anderer- biochemisch-physiologische Messungen beruhen meist auf der Vermessung von Mitochondrienpopulationen; zellbiologische Messungen zumeist auf der Erfassung einzelner, diskreter Organellen in Zellen oder Geweben. Zwar tauschen einzelne Mitochondrien durch Fusion und Teilung regelmäßig Komponenten aus; ihre Funktionen in der Zelle führen sie jedoch als diskrete Organisationseinheiten, also als Individuen, aus. Jedoch fehlt uns bisher ein quantitatives Verständnis davon, wie genau ein einzelnes Mitochondrium zusammengesetzt ist.

\section{Skalierung von Proteomdaten auf ein einzelnes Organell}

Eine einfache Annäherung kann erzielt werden, wenn man das Proteom von Mitochondrien (welches aus Proben bestimmt wird, die riesige Mengen einzelner Mitochondrien enthalten) auf die Dimensionen eines einzelnen Mitochondriums skaliert (Abb. 1). Für die quantitative Proteomanalyse verwendeten wir aufgereinigte Mitochondrien aus einer Zellkultur der Ackerschmalwand (Arabidopsis thaliana) [1]. Mithilfe eines labelfreien massenspektrometrischen shot gun-Ansatzes konnten wir die relativen

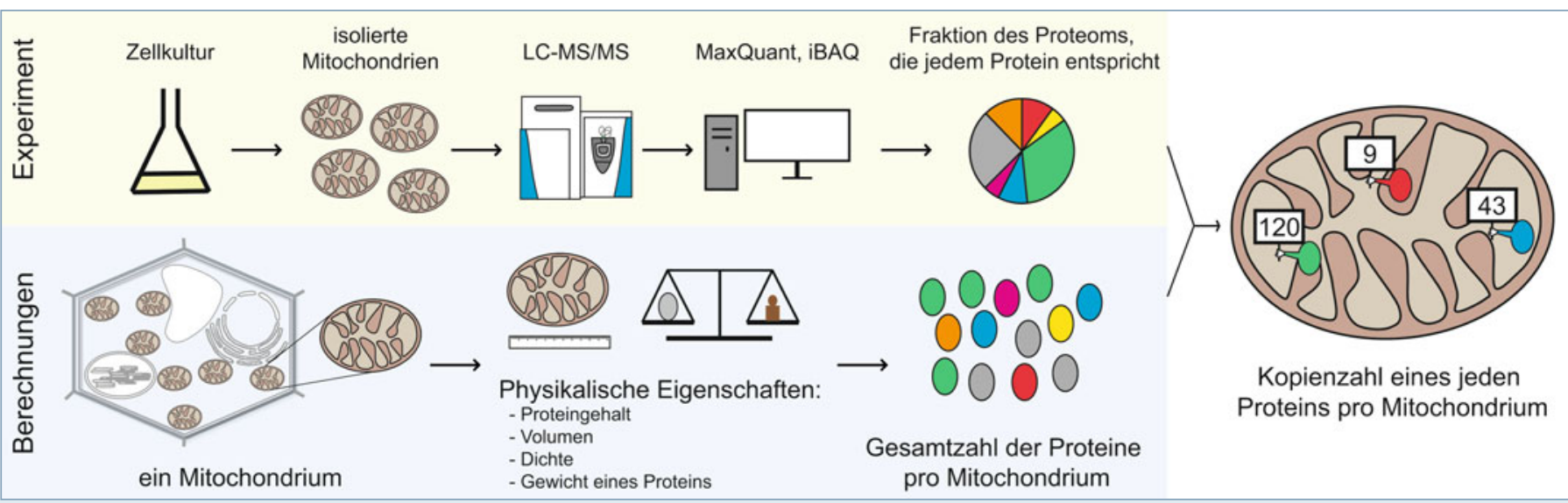

$\Delta$ Abb. 1: Abschätzung der Proteinausstattung eines einzelnen Mitochondriums mittels quantitativer Proteomik unter Berücksichtigung grundlegender physikalischer Eigenschaften eines einzelnen Mitochondriums von Arabidopsis thaliana. Die relativen Anteile der einzelnen Proteinspezies am Proteom isolierter Mitochondrien wurden durch shotgun-Massenspektrometrie anhand von iBAQ-Werten (intensity-based absolute quantification) experimentell bestimmt. Um die Kopienzahl eines jeden Proteins in einem einzelnen Mitochondrium zu berechnen, ist eine Abschätzung der Gesamtzahl vorhandener Proteinmoleküle erforderlich. Diese beruht auf dem Volumen und der Dichte eines repräsentativen Mitochondriums sowie seinem Proteingehalt und der durchschnittlichen Proteinmasse. 


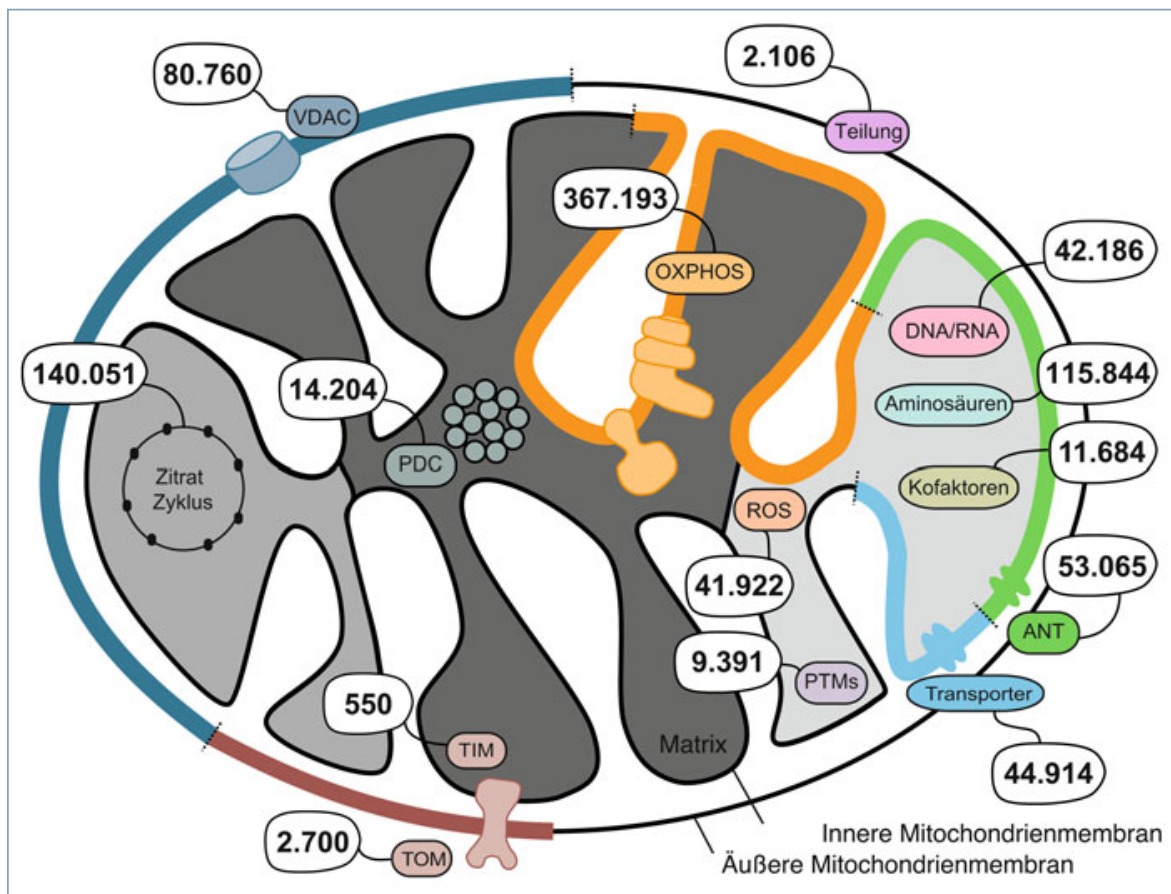

$\triangle$ Abb. 2: Die Proteinausstattung eines Mitochondriums. Ein repräsentatives Mitochondrium aus Arabidopsis-Zellkultur enthält insgesamt etwa 1,4 Millionen Proteinmoleküle. Ihre Verteilung auf die verschiedenen Stoffwechselwege wird hier anhand der aufsummierten Kopienzahlen aller beteiligten Proteine dargestellt. Eingefärbte Flächen illustrieren den Volumenanteil dieser Proteine an den Mitochondrienmembranen (verschiedene Farben) bzw. dem Gesamtproteom der Matrix (Graustufen), die zu 20 Prozent aus Proteinen besteht. Aminosäuren: Enzyme des Aminosäurestoffwechsels; ANT: ATP/ADP-Translokase; Kofaktoren: Biosynthese von Kofaktoren; DNA/RNA: mit dem mitochondriellen Genom assoziierte Proteine; OXPHOS: Komplexe der Atmungskette und der ATP-Synthasekomplex; PDC: Pyruvatdehydrogenasekomplex; PTM: posttranslationale Modifikationen (regulatorische Proteine); ROS: antioxidative Enzyme; Citratzyklus: Enzyme des Citratzyklus; TIM: translocase of the inner membrane (Anzahl der aktiven Dimere); TOM: translocase of the outer membrane (Anzahl der Trimere); Transporter: weitere Transporter der inneren Mitochondrienmembran (außer ANT); VDAC: voltage dependent anion channel.

Anteile der einzelnen Proteinspezies am Gesamtproteom abschätzen. Für die Skalierung der relativen Anteile benötigt man die Gesamtzahl aller Proteinmoleküle in einem einzelnen Mitochondrium. Diese Zahl lässt sich aus den physikalischen Eigenschaften eines repräsentativen Mitochondriums herleiten - wie anteiligem Proteingehalt, Volumen, Dichte und der durchschnittlichen Masse eines mitochondriellen Proteins. Für dieses Mitochondrium berechneten wir dann aus den Proteomdaten die Anzahl der enthaltenen Moleküle für jedes identifizierte Protein. Wichtig bei dieser Betrachtung ist, dass sowohl die Quantifizierung der Proteinanteile als auch deren Skalierung auf experimentellen Messwerten und Annahmen beruhen, die zwar sinnvoll sind, aber auch Unzulänglichkeiten enthalten. Mit dem Wissen um diese Limitierung lassen sich auf Basis der errechneten Kopienzahlen und bekannter Eigenschaften der einzelnen Proteine Gedankenexperimente durchführen, die faszinie- rende Einblicke in die Funktionsweise der Mitochondrien ermöglichen.

\section{Wie viele Kopien von welchem Protein enthält ein einzelnes Mitochondrium?}

Ein repräsentatives Mitochondrium enthält 1,4 Millionen einzelne Proteinmoleküle (Abb. 2). Ähnliche Berechnungen ergeben für eine Blatt-Mesophyllzelle eine Ausstattung von 25 Milliarden Proteinmolekülen, von denen sich 20 Milliarden auf die Chloroplasten und knapp 500 Millionen auf alle Mitochondrien verteilen [2]. Auf Grundlage dieser Zahlen lassen sich konkrete Gedankenexperimente zu Aufbau und Funktion eines Mitochondriums durchführen. Bei diesem gedanklichen Rundgang stechen die an der Produktion und dem Transport von ATP beteiligten Proteine durch ihre Dominanz in Masse und Häufigkeit hervor. Die ATP-Produktion erfordert eine vergleichsweise kleine Anzahl verschiedener Proteine, diese jedoch in jeweils hoher Kopienzahl. Die mitochondrielle Atmungskette nimmt mit insgesamt knapp 370.000 einzelnen Proteinmolekülen, die zu 20.000 Komplexen zusammengeschlossen sind, allein 18 Prozent der Oberfläche der inneren Mitochondrienmembran ein und bestimmt damit maßgeblich ihre Struktur [3]. Weitere 9,5 Prozent der inneren Mitochondrienmembran sind von knapp 98.000 Metabolittransportermolekülen besetzt, welche die Verbindung zur umgebenden Zelle herstellen. Das häufigste Mitochondrienprotein überhaupt ist mit 44.000 Kopien das Protein VDAC1 (voltage dependent anion channel 1), das zusammen mit den weiteren vier VDAC-Isoformen 34 Prozent der äußeren Mitochondrienmembran einnimmt und diese weitestgehend durchlässig für niedermolekulare Stoffe macht. Zum Vergleich: Die große Untereinheit von RubisCO, kommt in einer durchschnittlichen Blattzelle etwa 3,2 Milliarden mal vor, was etwa 32 Millionen Kopien in jedem Chloroplasten entspricht [2]. Mitochondrien synthetisieren nur wenige Proteine selbst, sind also auf den Import eines Großteils ihrer Proteinausstattung aus dem Cytosol angewiesen. Limitierend ist in diesem Fall die Präprotein-Translokase in der inneren Mitochondrienmembran, der TIMKomplex, der Proteine über die innere Mitochondrienmembran transportiert. Einzelnen Mitochondrien stehen 550 Kopien dieser dimeren Translokase zur Verfügung, wohingegen die Präprotein-Translokase der äußeren Mitochondrienmembran, der TOM-Komplex, mit 2.700 trimeren Kopien pro Mitochondrium vertreten ist. Die Transportkapazität würde dennoch ausreichen, um den Proteingehalt der Matrix innerhalb von weniger als sieben Stunden zu verdoppeln. Das Volumen der mitochondrialen Matrix wird unserer groben Abschätzung nach zu etwa 20 Prozent mit Proteinen ausgefüllt, ohne Berücksichtigung der sie umgebenden Hydrathüllen.

Die Ausstattung des repräsentativen Mitochondriums zeigt eine klare Dominanz des sehr großen Pyruvatdehydrogenasekomplexes, der mit insgesamt 14.200 einzelnen Proteinmolekülen etwa 50 Prozent des Proteinvolumens in der Mitochondrienmatrix ausmacht. Der Citratzyklus pflanzlicher Mitochondrien passt seine Funktionsweise den unterschiedlichen Anforderungen der Zelle flexibel an [4]. Er besteht aus neun Enzymen, die zusammen 140.000 Proteinmoleküle stellen. Pflanzliche Mitochondrien nutzen meist 
Malat als Substrat und besitzen die erforderliche Enzymausstattung, um innerhalb von einer Stunde ihre eigene Masse an Malat umzusetzen. Neben der Energieversorgung erfüllen Mitochondrien weitere essenzielle Stoffwechselfunktionen. Dementsprechend enthalten sie Enzyme für die Synthese von Kofaktoren und Eisen-Schwefel-Clustern, den Abbau von Aminosäuren und die Entgiftung reaktiver Sauerstoffspezies (ROS). Letztere werden offenbar vorsorglich im Überschuss vorgehalten. Die Kapazität der antioxidativen Enzyme eines Mitochondriums übertrifft die zu erwartende ROS-Produktion selbst unter Stressbedingungen bei Weitem. Zur Regulation der hoch abundanten Pyruvatdehydrogenase steht dagegen eine vergleichsweise geringe Anzahl regulatorischer Proteine zur Verfügung. So würden die 120 Kopien der PyruvatdehydrogenaseKinase eines Mitochondriums bei maximaler Aktivität eine knappe halbe Stunde benötigen, um alle 9.000 Moleküle ihres Substrates (der PDH-E1 $\beta$ Untereinheit) zu phosphorylieren, was in etwa der Zeitspanne entspricht, die für die Inaktivierung des Pyruvatdehydrogenasekomplexes im Licht beobachtet wurde [5]. Einzelne Faktoren, die am Edieren von mRNA beteiligt sind, liegen im Durchschnitt sogar mit weniger als einer Kopie pro Mitochondrium vor. Das bedeutet, dass nicht jedes Mitochondrium gleichzeitig über dieses Protein verfügen kann. Solche Proteine werden vermutlich zwischen einzelnen Mitochondrien durch regelmäßige Fusion und Teilung weitergegeben. Diese Schlussfolgerung entspricht der Beobachtung, dass auch die mitochondrielle DNA von Arabidopsis in substöchiometrischen Konzentrationen mit durchschnittlich nur einem vollständigen mitochondrialen Genom pro drei Mitochondrien vorliegt [6].

\section{Wie heterogen und wandelbar sind Mitochondrien?}

Für unsere Berechnungen haben wir ein repräsentatives Mitochondrium zugrunde gelegt. Eine solche Mittelung kann der eigentlichen Heterogenität der Mitochondrien natürlich nicht gerecht werden. In Samenpflanzen, die aus spezialisierten Zelltypen, Geweben und Organen aufgebaut sind und ihren Zell- stoffwechsel flexibel an wechselnde Umweltbedingungen anpassen können ist es augenscheinlich, dass die Komposition und Funktion der Mitochondrien diese Spezialisierung und Anpassung reflektieren (Abb. 3). Unterschiede in der Zusammensetzung der Proteome wurden bisher für Mitochondrienextrakte von pflanzlichen Strukturen gezeigt, die sich einfach mechanisch trennen lassen (Blätter, Wurzeln, Blüten, Stamm, Schoten, Zellkultur [7]). Jedoch ist dies nur die Spitze des Eisbergs, denn die Organe bestehen wiederum aus höchst unterschiedlichen Geweben und Zelltypen. Selbst auf Einzelzellebene ist es wahrscheinlich, dass die einzelnen Mitochondrien über unterschiedliche Proteinausstattungen verfügen (Abb. 3), wie es für spezifische Proteine bereits gezeigt werden konnte [8, 9]. Beispielsweise ist eine gezielte Anreicherung einzelner Mitochondrien mit bestimmten Proteinen wahrscheinlich - in Abhängigkeit von ihrer Positionierung in der Zelle und damit ihrer Funktion und ihrer Interaktion mit anderen Zellstrukturen. Um zukünftig ein realistisches und quantitatives Verständnis von Heterogenität mitochondrieller Strukturen und Funktionen zu erlangen, werden analytische Ansätze notwendig sein, die eine Mittelung von Unterschieden vermeiden. Genetische Tagging-Methoden bieten bereits die Möglichkeit, Mitochondrien aus definierten Zelltypen aufzureinigen [10-13] und so Populationen gezielt zu trennen. Zukünftig wäre eine direkte Untersuchung der Proteinausstattung einzelner Mitochondrien wünschenswert, wie sie sich in der Entwicklung spezialisierter bildgebender Techniken (z. B. Kryoelektronentomographie) oder hochauflösender massenspektroskopischer Verfahren (z. B. MALDI-Imaging, Single Cell Proteomics) andeutet.

\section{Danksagung}

Wir danken allen Autoren der hier beleuchteten Studie. Der Austausch und die gemeinsamen Arbeiten der Plant Mito Group wurden in Teilen durch die Deutsche Botanische Gesellschaft und die Deutsche Forschungsgemeinschaft unterstützt (PAK918 - EU54/4-1, IF1655/3-1, MA2379/14-1, MU4137/11, SCHA1953/3-1, SCHW1719/5-1). 

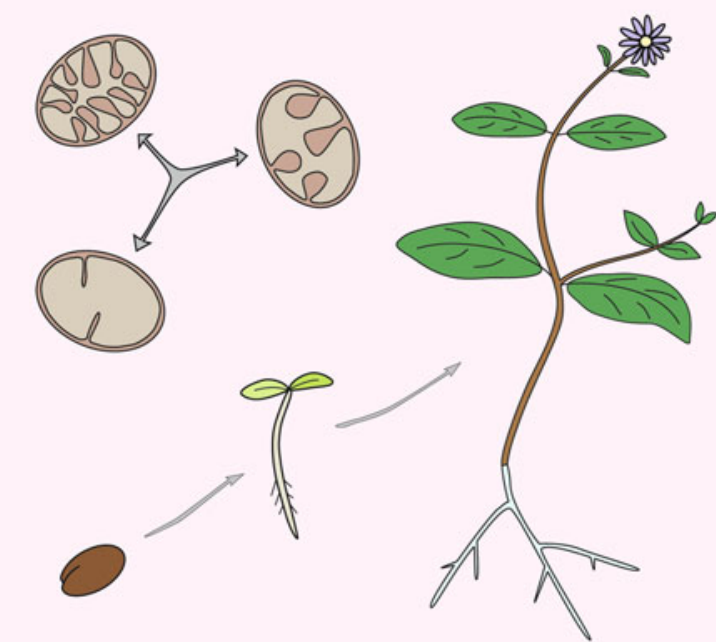

\section{Spezialisierung nach Zelltyp und Gewebe}

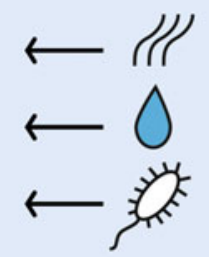

\section{Akklimatisierung an Umweltfaktoren}

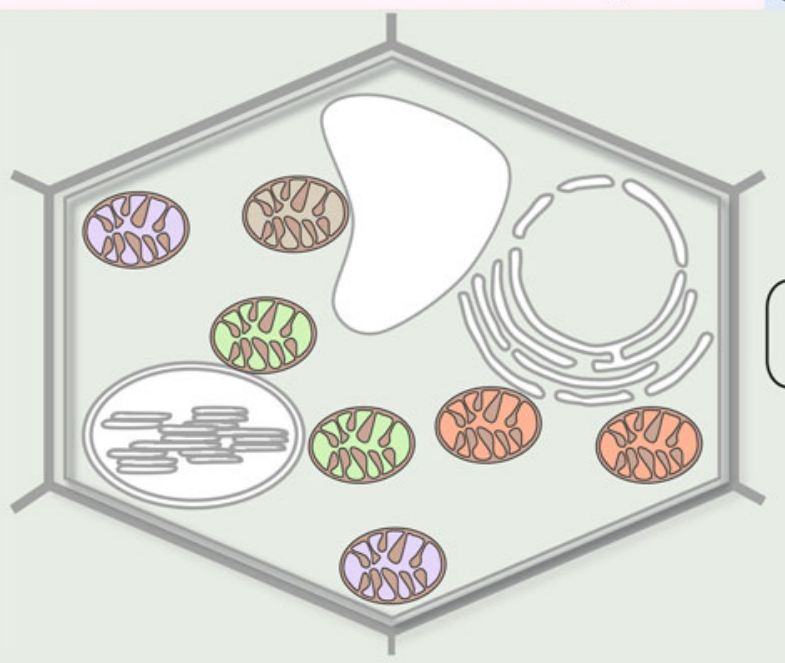

\section{Spezialisierung innerhalb der Zelle}

$\Delta$ Abb. 3: Eine wichtige zukünftige Herausforderung wird darin bestehen, die proteomischen Unterschiede zwischen Mitochondrien und deren Plastizität experimentell zu analysieren. Heterogenität ist dabei nicht nur in der Entwicklung, zwischen Organen, Geweben und Zelltypen zu erwarten, sondern auch zwischen den Mitochondrien in einzelnen Zellen. Hinzu kommt die flexible Anpassung der Proteinausstattung als Antwort auf Umweltfaktoren. Ein realistisches und umfassendes Verständnis bedarf zukünftig der Vermessung dieser quantitativen Unterschiede.
[9] Fuchs R, Kopischke M, Klapprodt C et al. (2016) Immobilized subpopulations of leaf epidermal mitochondria mediate PENETRATION2-dependent pathogen entry control in Arabidopsis. Plant Cell 28: 130-145

[10] Kuhnert F, Stefanski A Overbeck N et al. (2020) Rapid single-step affinity purification of HA-tagged plant mitochondria. Plant Physiol 182: 692-706

[11] Boussardon C, Przybyla-Toscano J, Carrie C, Keech O (2020) Tissue-specific isolation of Arabidopsis/plant mitochondria - IMTACT (isolation of mitochondria tagged in specific cell types). Plant J 103: 459-473

[12] Niehaus M, Straube H, Künzler P et al. (2020) Rapid affinity purification of tagged plant mitochondria (Mito-AP) for metabolome and proteome analyses. Plant Physiol 182: 1194-1210

[13] Lang M, Pröschel M, Brüggen N, Sonnewald U (2020) Tagging and catching: rapid isolation and efficient labeling of organelles using the covalent Spy-System in planta. Plant Methods 16: 122

Funding note: Open Access funding enabled and organized by Projekt DEAL Open Access: Dieser Artikel wird unter der Creative Commons Namensnennun 4.0 International Lizenz veröffentlicht, welche die Nutzung, Vervielfältigung, Bearbeitung, Verbreitung und Wiedergabe in jeglichem Medium und Forma erlaubt, sofern Sie den/die ursprünglichen Autor(en) und die Quelle ordnungsgemäß nennen, einen Link zur Creative Commons Lizenz beifügen und angeben, ob Änderungen vorgenommen wurden. Die in diesem Artikel enthaltenen Bilder und sonstiges Drittmaterial unterliegen ebenfalls der genannten Creative Commons Lizenz, sofern sich aus der Abbildungslegen

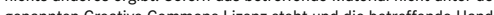
gench Weiterverwendungen des Materials die Einwilligung des jeweiligen Rechteinhabers einzuholen. Weitere Details zur Lizenz entnehmen Sie bitte der Lizenzinformation auf http://creativecommons.org/licenses/by/4.0/deed.de.

\section{Korrespondenzadresse:}

Dr. Etienne Meyer

Institutsbereich Pflanzenphysiologie, Abteilung Zellphysiologie

Martin-Luther-Universität Halle-Wittenberg

Weinbergweg 10

D-06120 Halle (Saale)

etienne.meyer@pflanzenphys.uni-halle.de https://blogs.urz.uni-halle.de/cellphysiology

\section{Literatur}

[1] Fuchs P, Rugen N, Carrie C et al. (2020) Single organelle function and organization as estimated from Arabidopsis mitochondrial proteomics. Plant J 101: 420-441

[2] Heinemann B, Künzler P, Eubel H et al. (2021) Estimating the number of protein molecules in a plant cell: protein and amino acid homeostasis during drought. Plant Physiol 185 385-404

[3] Dudkina NV, Heinemeyer J, Keegstra W et al. (2005) Structure of dimeric ATP synthase from mitochondria: an angular association of monomers induces the strong curvature of the inner membrane. FEBS Lett 579: 5769-5772 [4] Sweetlove LJ, Beard KF, Nunes-Nesi A et al. (2010) Not just a circle: flux modes in the plant TCA cycle. Trends Plant Sci 15: 462-470

[5] Budde RJ, Randall DD (1990) Pea leaf mitochondrial pyruvate dehydrogenase complex is inactivated in vivo in a light-dependent manner. Proc Natl Acad Sci USA 87: 673676

[6] Preuten T, Cincu E, Fuchs J et al. (2010) Fewer genes than organelles: extremely low and variable gene copy numbers in mitochondria of somatic plant cells. Plant J 64: 948-959

[7] Lee CP, Eubel H, Solheim C, Millar AH (2012)

Mitochondrial proteome heterogeneity between tissues from the vegetative and reproductive stages of Arabidopsis thalia na development. J Proteome Res 11: 3326-3343

[8] Wurm CA, Neumann D, Lauterbach MA et al. (2011) Nanoscale distribution of mitochondrial import receptor Tom20 is adjusted to cellular conditions and exhibits an inner-cellular gradient. Proc Natl Acad Sci USA 108: 1354613551

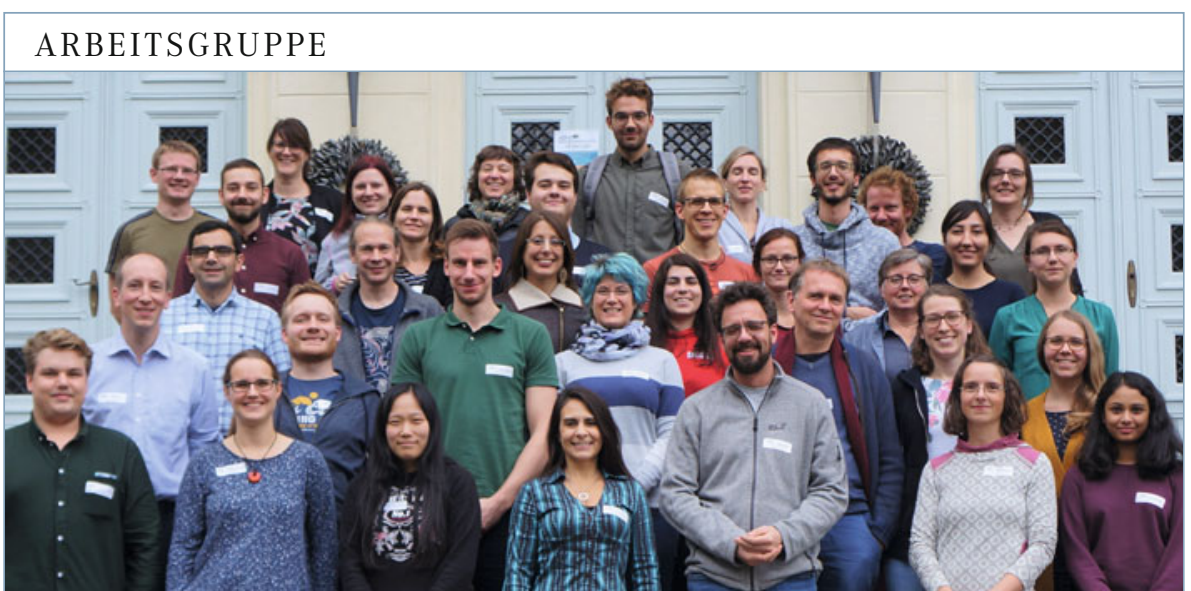

Treffen der Plant Mito Group in Düsseldorf, 2018.

Das hier diskutierte proteombasierte Gedankenexperiment ist ein Resultat der gemeinsamen Aktivitäten der Plant Mito Group als informelle, offene Plattform von Wissenschaftler:innen in Deutschland (bisher aus Berlin, Bonn, Bremen, Düsseldorf, Halle, Hannover, Kaiserslautern, München, Münster, Potsdam und UIm), die sich für die unterschiedlichsten Aspekte mitochondrieller Biologie in Pflanzen begeistern. Seitdem die Idee für diese Gruppe 2013 am Rande der International Conference for Plant Mitochondria in Rosario (Argentinien) u. a. von Axel Brennicke (1953-2017) angestoßen wurde, finden halbjährliche Treffen zur Diskussion aktueller Fragen um Mitochondrien der Pflanzen statt. 